\title{
EXPERIMENTAL INVESTIGATION OF DROPLET EVAPORATION OF WATER WITH GROUND ADMIXTURES WHILE MOTION IN A FLAME OF LIQUID FUEL
}

\author{
Margarita A. Dmitriyenko ${ }^{1}$, Galina S. Nyashina ${ }^{1}$, Alena O. Zhdanova ${ }^{1}$, Olga V. Vysokomornaya ${ }^{1}$ a \\ ${ }^{1}$ National Research Tomsk Polytechnic University, 634050 Tomsk, Russia
}

\begin{abstract}
The evaporation features for the atomized flow of suspension on the base of water with ground admixtures in an area of high-temperature combustion products of liquid flammable substance (acetone) were investigated experimentally by the optical methods of gas flow diagnostic and the high-speed video recording. The scales of influence of clay and silt concentration in droplets of atomized flow on the intensity of its evaporation were determined. The approximation dependences describing a decrease in typical size of suspension droplets at various values of ground admixtures were obtained.
\end{abstract}

\section{Introduction}

Evaporation of flows of atomized liquids in high-temperature gas mediums is a widespread technological process in many industries. As process liquids in such processes, it is often used water and also solutes, emulsions and suspensions on its base: in power engineering at forming gas-vapor heat carriers [1], at polydisperse fire extinguishing [2], at the high-temperature purification of industrial and effluent waters [3].

Completeness and the rate of evaporation of atomized liquids in these technologies is largely determined by the characteristic dimensions of the droplets [4]. However, it was presented in the work [5] that if the value of the typical size of atomized liquid flow droplets was less than $100 \mu \mathrm{m}$, it was possible the deceleration, turning and taking away a liquid by the flow of gas medium moving in the opposite direction, from whence the intensity and completeness of evaporation were decreased. In the alternative of forming the liquid flow with droplet sizes less than $100 \mu \mathrm{m}$ we can consider injecting into droplets the solid particles, the thermophysical and optical properties of which would contribute to more rapid heating up of particles [5]. While the formation of liquid flow based on water, for the identified properties, on the one hand, it can be used wide-spread ground admixtures (clay, silt and other), and on the other - chemically inert ones.

The purpose of the work is to determine experimentally the integral characteristics of droplet evaporation of atomized suspension based on water with typical ground admixtures in a flame of flammable liquid.

\section{Experimental setup and procedure}

\section{a Corresponding author: vysokomornaja@tpu.ru}


In experimentations we used the setup similar to that used in the work [6]. To investigate the processes of evaporation of atomized composition (water with ground admixtures) while moving through high-temperature gas medium, it was applied the equipment of cross-correlation complex [6]: sync processor, double pulse $\mathrm{Nd}$ :YAG laser (wavelength $-532 \cdot 10^{-9} \mathrm{~m}$, lowest energy in pulse $70 \cdot 10^{-3} \mathrm{~J}$, maximum pulse time $-12 \cdot 10^{-9} \mathrm{~s}$, repetition frequency $-15 \mathrm{~Hz}$ ), generator of laser emission, two CCD video cameras (picture size $-2048 \times 2048$ pixels, frame frequency $-1.5 \mathrm{~Hz}$, minimum delay between frames $\left.-5 \cdot 10^{-6} \mathrm{~s}\right)$.

To the output of the container containing the previously prepared suspension based on water and ground elements, we connected through the channel an atomizer, which was configured according to the required atomization parameters. The base of hollow cylinder was filled with liquid flammable substance (acetone), ignition of which was initiated before the experimentation. When the temperature of combustion products $T_{\mathrm{g}}$ reached the value about $1100 \mathrm{~K}$, we activated the atomizer fastened at a distance of $0.5 \mathrm{~m}$ from the upper face of the cylinder. The specialized software (it realizes the optical methods PIV, PTV, IPI or SP) [7] was started on the PC, and video recording of motion of atomized suspension flow was performed (shooting was carried out at the inlet and outlet of the cylindrical channel). To estimate the completeness of suspension droplet evaporation while their passing through the high-temperature gas medium, we used the parameter $\Delta R$ characterizing the decrease of droplet sizes at motion through high-temperature gases $\left(\Delta R=\left(R_{\mathrm{m}}-R_{\mathrm{m}}{ }^{*}\right) / R_{\mathrm{m}}\right.$, where $R_{\mathrm{m}}, R_{\mathrm{m}}{ }^{*}$ is values of condition mean radius of droplet at the inlet and outlet from high-temperature gases, $\mathrm{mm}$ ).

\section{Results and Discussion}

Fig. 1 illustrates the dependences of $\Delta R$ parameter on mass concentration in droplets of atomized suspension of clay and silt.

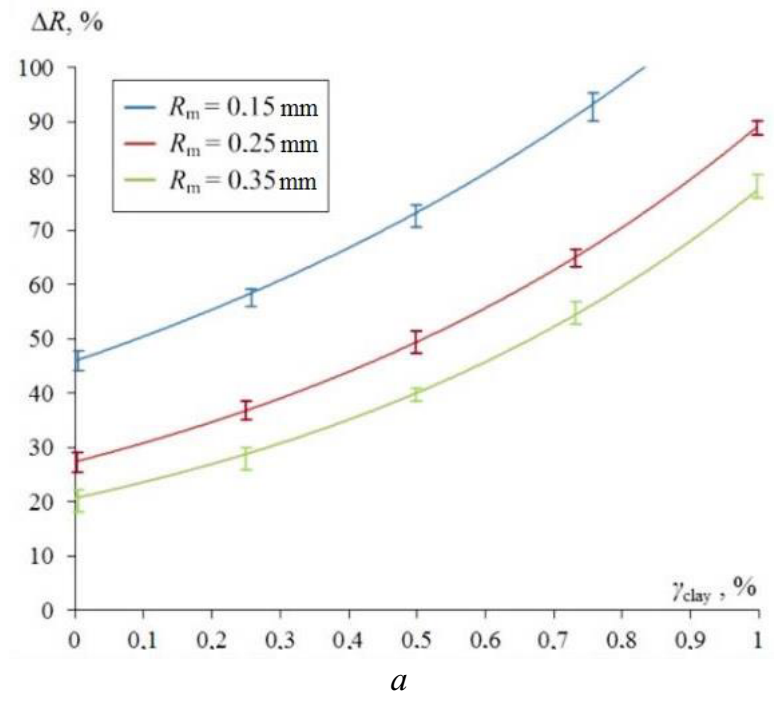




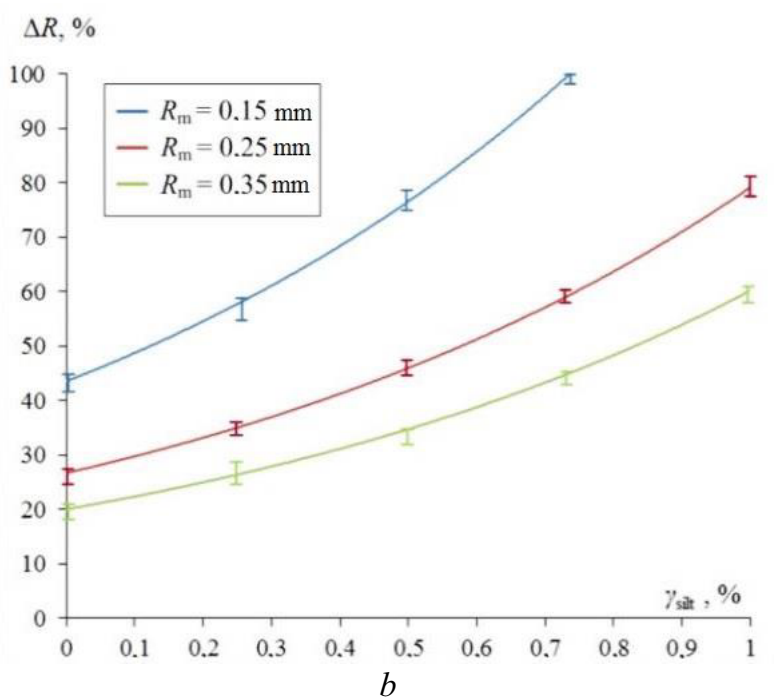

Figure 1. Dependences of $\Delta R$ parameter for various initial sizes $R_{\mathrm{m}}$ of atomized suspension droplets on the mass concentration of clay $(a)$ and silt $(b)$ admixtures in its composition while passing the distance of $1 \mathrm{~m}$ by droplets in a high-temperature $\left(T_{\mathrm{g}} \approx 1100 \mathrm{~K}\right)$ gas medium.

For the dependences presented in Fig. 1, we obtained approximation expressions characterizing the decrease of the radii of atomized droplets at various values of concentrations of ground admixtures in them.

For droplets with admixture of clay:

initial size $R_{\mathrm{m}} \approx 0.15 \mathrm{~mm}$

$\Delta R=45.959 \cdot \exp \left(0.9343 \cdot \gamma_{\text {clay }}\right), 0<\gamma_{\text {clay }}<1 \%, U_{\mathrm{m}} \approx 2 \mathrm{~m} / \mathrm{s}, T_{\mathrm{w}} \approx 278 \mathrm{~K}$;

initial size $R_{\mathrm{m}} \approx 0.25 \mathrm{~mm}$

$\Delta R=27.41 \cdot \exp \left(1.1815 \cdot \gamma_{\text {clay }}\right), 0<\gamma_{\text {clay }}<1 \%, U_{\mathrm{m}} \approx 2 \mathrm{~m} / \mathrm{s}, T_{\mathrm{w}} \approx 278 \mathrm{~K}$;

initial size $R_{\mathrm{m}} \approx 0.35 \mathrm{~mm}$

$\Delta R=20.71 \cdot \exp \left(1.3218 \cdot \gamma_{\text {clay }}\right), 0<\gamma_{\text {clay }}<1 \%, U_{\mathrm{m}} \approx 2 \mathrm{~m} / \mathrm{s}, T_{\mathrm{w}} \approx 278 \mathrm{~K}$.

For droplets with admixture of silt:

initial size $R_{\mathrm{m}} \approx 0.15 \mathrm{~mm}$

$\Delta R=43.549 \cdot \exp \left(1.1285 \cdot \gamma_{\text {silt }}\right), 0<\gamma_{\text {silt }}<1 \%, U_{\mathrm{m}} \approx 2 \mathrm{~m} / \mathrm{s}, T_{\mathrm{w}} \approx 278 \mathrm{~K}$;

initial size $R_{\mathrm{m}} \approx 0.25 \mathrm{~mm}$

$\Delta R=26.711 \cdot \exp \left(1.0862 \cdot \gamma_{\text {silt }}\right), 0<\gamma_{\text {silt }}<1 \%, U_{\mathrm{m}} \approx 2 \mathrm{~m} / \mathrm{s}, T_{\mathrm{w}} \approx 278 \mathrm{~K}$;

initial size $R_{\mathrm{m}} \approx 0.35 \mathrm{~mm}$

$\Delta R=20.069 \cdot \exp \left(1.0986 \cdot \gamma_{\text {silt }}\right), 0<\gamma_{\text {silt }}<1 \%, U_{\mathrm{m}} \approx 2 \mathrm{~m} / \mathrm{s}, T_{\mathrm{w}} \approx 278 \mathrm{~K}$.

Analyzing the dependences presented in Fig. 1, it can be concluded that the presence of ground admixtures in droplets of atomized suspensions led to significant intensification of heat and mass transfer in high-temperature (about $1100 \mathrm{~K}$ ) combustion products. If droplets with typical sizes $R_{\mathrm{m}}=0.15 \mathrm{~mm}$ passed the distance of $1 \mathrm{~m}$, they evaporated completely at a mass concentration of clay and silt $\gamma_{\text {clay }}=85 \%$ and $\gamma_{\text {silt }}=70 \%$ correspondingly. Parameter $\Delta R$ for droplets of "pure" water (without ground admixtures) under similar conditions $\left(R_{\mathrm{m}}=0.15 \mathrm{~mm}\right.$, temperature of the combustion products of liquid fuel $T_{\mathrm{g}} \approx 1100 \mathrm{~K}$, the distance passed in a medium of hot gases) was about $45 \%$.

Differences in thermophysical and optical characteristics of the suspension and water without admixtures can explain the obtained effect. Suspension droplets are generated the energy of thermal and optical radiation of combustion products more intensive, from whence there is a faster (relatively to water without admixtures) heat of the droplet. Therefore, ground elements absorb and accumulate the high-temperature gas medium energy supplied by the water layer (creating additional local heating sources inside the droplets, that is at the internal borders of the interface) more intensive by 5-7 times 
on the average. Thus, it confirms the applicability of compositions on the base of water with solid ground admixtures in polydisperse fire extinguishing, producing technologies of two-phase gas-vapor heat carriers and other, as well as efficiency of thermal deactivation of process water containing, inter alia, the elements of the ground and various nontransparent solutions.

\section{Conclusions}

The results of experimental investigations in determining the evaporation characteristics of the atomized flow of suspension based on water with ground admixtures in high-temperature (more than $1100 \mathrm{~K}$ ) combustion products of organic liquid allow concluding about intensification of heat and mass transfer and phase transformation due to the presence suspensions of solid lightproof ground particles in droplets. The obtained dependences of characteristic droplet size $\Delta R$ decrease show strengthening this effect by increasing the concentration of the ground admixture in water droplets.

\section{Acknowledgments}

The study was supported by the Russian Foundation for Basic Research (project 14-08-00057). The analysis of the influence of the typical size of suspension droplets on their evaporation conditions was performed with financial support of the scholarship of the President of the Russian Federation for young scientists (SP-1350.2015.1).

\section{References}

1. M.N. Nikitin, Promyshlennaya Energetika (Power Industry). 6 (2010) (in Russian).

2. Z. Tang, Z. Fang, J.P. Yuan, B. Merci, Fire Safety J. 55 (2013).

3. V.B. Tulepbayev, I.Yu. Dyatchenko, Gal'vanotechnika I obrabotka poverhnosti (Electroplating and surface treatment) 16, 1 (2008) (in Russian).

4. G.V. Kuznetsov, P.A. Strizhak, Tech. Phys. Lett. 40, 6 (2014).

5. R.S. Volkov, G.V. Kuznetsov, P.A. Strizhak, Int. J. Heat and Mass Trans. 79 (2014).

6. A.O. Zhdanova, O.V. Vysokomornaya, P.A. Strizhak, Procedia - Social and Behavioral Sciences 206 (2015).

7. S. Dehaeck, H. Van Parys, Hubin A., J.P.A.J. van Beeck, Exp. Fluids. 47 (2009). 